PROCEEDINGS OF THE

AMERICAN MATHEMATICAL SOCIETY

Volume 130, Number 5 , Pages 1539-1550

S 0002-9939(01)06184-6

Article electronically published on October 5, 2001

\title{
ON A THEOREM OF JAWOROWSKI ON LOCALLY EQUIVARIANT CONTRACTIBLE SPACES
}

\author{
SERGEI M. AGEEV AND DUŠAN REPOVŠ
}

(Communicated by Alan Dow)

\begin{abstract}
Ancel's method of fiberwise trivial relations is applied to the problem of characterization of absolute equivariant extensors. We obtain a generalization of Jaworowski's theorem on characterization of equivariant extensors lying in $\mathbb{R}^{n}$ to the case when the space is infinite-dimensional, has infinitely many orbit types and the acting compact group $G$ is not necessarily a Lie group.
\end{abstract}

\section{INTRODUCTION}

Spaces which possess absolute extension property for partially defined maps, are called absolute (neighborhood) extensors (denoted by $\mathrm{A}[\mathrm{N}] \mathrm{E}$ ) and they play an important role in topology. When a compact group $G$ acts on the space, similar objects with respect to $G$-maps are called equivariant absolute (neighborhood) extensors (denoted by $G-\mathrm{A}[\mathrm{N}] \mathrm{E}$ ). We recall that a metric space $X$ is said to be a $G$-AE (resp. $G$-ANE) space, if for every partial $G$-map $Z \supset A \stackrel{\varphi}{\rightarrow} X$, defined on a closed $G$-subspace $A$ of a metric $G$-space $Z$, there exists a $G$-extension $\widehat{\varphi}: Z \rightarrow X$ onto the entire space $Z$ (resp. $\widehat{\varphi}: U \rightarrow X$ onto a $G$-neighborhood $U$ of the subspace $A$ ). Observe that the following notions are identical:

(a) equivariant absolute (neighborhood) extensors and absolute (neighborhood) extensors for spaces with trivial $G$-action; and

(b) equivariant absolute (neighborhood) extensors and equivariant absolute (neighborhood) retracts for metric $G$-spaces.

The recognition of $G-\mathrm{A}[\mathrm{N}] \mathrm{E}$ is a difficult problem. One possible approach is to find sufficiently simple (for verification) properties which guarantee that $X \in G-\mathrm{A}[\mathrm{N}] \mathrm{E}$. Working in this direction, Jaworowski [Ja] proved a theorem on characterization of equivariant extensors lying in a finite-dimensional Euclidean space $\mathbb{R}^{n}$ with orthogonal action of a compact Lie group $G$ in terms of local $G$-contractibility, which can be reformulated as follows.

Theorem 1.1. Every invariant subspace $X \subset \mathbb{R}^{n}$ is a $G$-ANE (resp. $G$-AE) space if and only if $X$ is a locally $G$-contractible (resp. locally $G$-contractible and $G$ contractible) space.

Received by the editors May 17, 2000 and, in revised form, November 10, 2000.

1991 Mathematics Subject Classification. Primary 54C55, 57S10; Secondary 54C20, 54C60, $54 \mathrm{~F} 65,55 \mathrm{M} 15$.

Key words and phrases. Local equivariant contractibility, absolute equivariant extensor, fiberwise trivial relation, countable dimensional orbit space, selections of multivalued maps. 
We recall that a $G$-contractible space $X$ is by definition a space which has the $G$ homotopy type of a point (i.e. there exists a fixed point $x_{0} \in X^{G}$ and a $G$-homotopy $F_{t}: X \rightarrow X$ such that $F_{0}=$ Id and $\left.F_{1}(X)=x_{0}\right)$. Introducing the concept of local $G$-contractibility of a space is a more delicate problem even for a compact Lie group G.

By a theorem of Palais $[\mathrm{Br}]$ there exists a neighborhood $G$-retraction $r: U \rightarrow$ $G(x)$ onto an arbitrary orbit $G(x)$. Thus this invariant neighborhood $U$ of $G(x)$ is $G$-homeomorphic to the cross-product $G \times_{G_{x}} S$, where $S \rightleftharpoons r^{-1}(x) \subset U$ is a $G_{x}$-space (cf. $\left.[\mathrm{Br}]\right)$. This invariant neighborhood $U$ is called an exact tube around the orbit $G(x)$ and the $G_{x}$-space $S$ is called an exact slice at the point $x$.

We shall say that:

(c) $X$ is locally $G$-contractible at the point $x \in X^{G}$, if for every $\epsilon>0$ there exist an invariant neighborhood $\mathcal{O}(x)$ and a $G$-homotopy $F: \mathcal{O}(x) \rightarrow \mathrm{N}(x ; \epsilon)$ such that $F_{0}=\mathrm{Id}$ and $F_{1}(\mathcal{O}(x))=x$;

(d) $X$ is locally $G$-contractible at the point $x \notin X^{G}$ if the slice $S$ of every tube $\mathcal{U}=G \times_{G_{x}} S$ around the orbit $G(x)$ is locally $G_{x}$-contractible at the point $x \in S^{G_{x}}$.

The following theorem describes the local $G$-contractibility in different terms.

Theorem 1.2. Let $G$ be any compact Lie group and $X$ any metric $G$-space. Then the following conditions are equivalent:

(1) $X$ is a locally $G$-contractible space at every point;

(2) for every point $x \in X$ there exists a tube $\mathcal{U}=G \times_{G_{x}} S$ around the orbit $G(x)$ whose slice $S$ is locally $G_{x}$-contractible at $x$;

(3) for every orbit $G(x)$ and every $\epsilon>0$ there exist a $G$-neighborhood $\mathcal{U} \supset$ $G(x)$ and a G-homotopy $F_{t}: \mathcal{U} \rightarrow X$ such that $F_{0}=\operatorname{Id}, F_{1}(\mathcal{U}) \subset G(x)$ and $\bigcup\left\{F_{t}(x) \mid 0 \leq t \leq 1\right\} \subset \mathrm{N}(x ; \epsilon) ;$ and

(4) for every point $x$ and every $\epsilon>0$ there exists a $G_{x}$-neighborhood $\mathcal{U}$ of $x$ which is $G_{x}$-contractible to the point $x$ inside the neighborhood $N(x ; \epsilon)$.

Remarks. (i) The initial definition of local $G$-contractibility, which implicitly appears in Ja], agrees with the property (4) above.

(ii) This theorem fails beyond the class of Lie groups because there is no tubular structure of invariant neighborhoods [Vi]. A version of the concept of local $G$ contractibility for arbitrary compact groups will be given later.

Proof of Theorem 1.2 (2) $\Longrightarrow(3)$. For every $x$ and $\epsilon>0$, there exists a tube $\mathcal{V}=G \times_{G_{x}} S$ around $G(x)$ such that $S$ is locally $G_{x}$-contractible at $x$ and $S \subset$ $\mathrm{N}(x ; \epsilon)$. Hence, by $(2)$, there exists a thinner tube $\mathcal{V}_{1}=G \times_{G_{x}} S_{1}$ around $G(x)$, the slice $S_{1}$ of which admits a $G_{x}$-contraction $\Phi_{t}: S_{1} \rightarrow S$ into $x$. Then the desired $G$-neighborhood $\mathcal{U}$ coincides with $\mathcal{V}_{1}$ and the desired $G$-homotopy $F_{t}: \mathcal{V}_{1} \rightarrow X$ is defined by the formula $F_{t}\left(\left[g, s_{1}\right]\right)=g \cdot \Phi_{t}\left(s_{1}\right)$.

$(3) \Longrightarrow(4)$. Fix $x$ and $\epsilon>0$. Since the smooth manifold $G(x)$ belongs to $G_{x}$-ANE, there exists $\delta>0$ such that $\mathrm{N}(x ; \delta) \cap G(x)$ admits a $G_{x}$-contraction in $x$ inside $\mathrm{N}(x ; \epsilon) \cap G(x)$. By (3), there exist a tube $\mathcal{U}=G \times_{G_{x}} S$ around $G(x)$ and a $G$-homotopy $\Phi_{t}: \mathcal{U} \rightarrow X$, such that $\Phi_{0}=\operatorname{Id}, \Phi_{1}(\mathcal{U}) \subset G(x)$ and $\bigcup\left\{\Phi_{t}(x) \mid 0 \leq t \leq\right.$ $1\} \subset \mathrm{N}(x ; \delta / 2)$. By reducing the tube $\mathcal{U}$, we can suppose that $\bigcup\left\{\Phi_{t}(\mathcal{W}) \mid 0 \leq t \leq\right.$ $1\} \subset \mathrm{N}(x ; \delta)$, where $\mathcal{W}$ is a fine $G_{x}$-neighborhood of $S$ in $\mathcal{U}$. Then the desired $G_{x^{-}}$ contraction $\mathcal{W}$ in $x$ inside $\mathrm{N}(x ; \epsilon)$ is defined in two steps: first, $G_{x}$-homotopy $F_{t} \rightleftharpoons$ 
$\Phi_{t}\left\lceil\mathcal{W}: \mathcal{W} \rightarrow \mathrm{N}(x ; \delta) \quad G_{x}\right.$-contracts $\mathcal{W}$ into $\mathrm{N}(x ; \delta) \cap G(x)$, and then $\mathrm{N}(x ; \delta) \cap G(x)$ is $G_{x}$-contracted in $x$ inside $\mathrm{N}(x ; \epsilon) \cap G(x)$.

$(4) \Longrightarrow(1)$. Consider an arbitrary tube $\mathcal{V}=G \times_{G_{x}} S$ around $G(x)$ and fix some $G_{x}$-retraction $r: \mathcal{W} \rightarrow S$ of a $G_{x}$-neighborhood $\mathcal{W} \subset \mathcal{V}$ onto $S$ which exists by the following assertion (this is an easy consequence of $[\mathrm{Mu}]$ ).

Lemma 1.3. Let $\mathcal{U}=G \times_{G_{x}} S$ be any tube around $G(x)$. Then $S$ is a neighborhood $G_{x}$-retract of $\mathcal{U}$.

Proof. Since $G_{x}$ is $G_{x}$-ANE, it follows that $G_{x}$ is a $G_{x}$-retract of some $G_{x}$-neighborhood $\mathcal{W}^{\prime} \subset G: r^{\prime}: \mathcal{W}^{\prime} \rightarrow G_{x}, r^{\prime} \circ r^{\prime}=r^{\prime}$. Then the desired $G_{x}$-retraction $r: \mathcal{W} \rightarrow$ $S$ of $G_{x}$-neighborhood $\mathcal{W} \rightleftharpoons \mathcal{W}^{\prime} \times{ }_{G_{x}} S$ of the slice $S$ is defined by the formula $r([w, s])=\left[r^{\prime}(w), s\right], w \in \mathcal{W}^{\prime}, s \in S$.

We now complete the proof of Theorem 1.2. We derive from (4) that for $\epsilon>0$, such that $\mathrm{N}(x ; \epsilon) \subset \mathcal{W}$, there exist a $G_{x}$-neighborhood $\mathcal{U} \ni x$ and a $G_{x}$-contraction $\Phi_{t}: \mathcal{U} \rightarrow \mathrm{N}(x ; \epsilon)$ to the point $x$. Let the tube $\mathcal{V}_{1}=G \times_{G_{x}} S_{1}, S_{1} \subset S$, be so small that $S_{1} \subset \mathcal{U}$. Then the desired $G_{x}$-contraction $F_{t}: S_{1} \rightarrow S$ is defined by the formula $F_{t}=r \circ \Phi_{t}$.

When the group action is trivial the concept of $G$-LC agrees with the local contractibility (LC). Next, with respect to simplicity, an example of a $G$-LC-space is a linear $G$-space. Since the $G$-LC property can be easily detected and since it is well-known that $G$-ANE $\subset G$-LC, there is an interesting question as to what degree is the local $G$-contractibility of a space characterized by its $G$-extensor properties. We observe that if the group action is trivial, the implication $G$-LC $\Longrightarrow G$-ANE is true under some dimensional restrictions on $X$ ( $X$ is finite-dimensional - the Kuratowski-Dugundji theorem $[\mathrm{Hu}], X$ is countable dimensional-Haver's theorem $\mathrm{Ha}$ ). Theorem 1.1 can be considered as a partial extension of the KuratowskiDugundji theorem to the equivariant case.

By the Mostow theorem, a separable metric $G$-space $X$ equivariantly lying in $\mathbb{R}^{n}$ can be characterized by the property that $X$ has finitely many orbit types and $\operatorname{dim} X<\infty$. Both of these properties are essentially applied in the characterization of equivariant extensors lying in $\mathbb{R}^{n} \mathrm{Ja}$. The present paper is a result of our efforts to understand what happens with this problem when the space $X \in G$-LC is infinite-dimensional and has infinitely many orbit types and the acting group $G$ is not necessarily a Lie group. As a result we have the following theorem.

Theorem 1.4. Let $G$ be any compact group and suppose that $X \in G$-LC. Then $X \in G$-ANE, provided that $X / G$ is a countable-dimensional space.

Remarks. (iii) If the acting group is trivial, then Theorem 1.4 coincides with the Haver theorem [Ha].

(iv) Theorem 1.4 is valid, provided that the orbit space $X / G$ satisfies a more general condition of being a $C$-space (see [AG] and [An, Appendix]).

(v) Beyond the class of compact groups Theorem 1.4 admits the generalization to so-called proper actions of locally compact Lie groups in the sense of Palais.

The second consequence is related to the problem of whether a linear metric $G$-space $L$ belongs to the class $G$-AE. Below we list known affirmative results.

(vi) $\operatorname{dim} L / G<\infty$ (the proof of this case is easily reduced to the case of a compact Lie group acting on a finite-dimensional space with the orthogonal representation); or 
(vii) $L$ is countable-dimensional and $|G|=1$ (this is a consequence of the Haver theorem); or

(viii) $L$ is locally convex and $L \in G$-ANE(0) $\mathrm{Ag}$ (in particular, $G$ is a compact Lie group [Mu, p. 489] or $L$ is a Banach $G$-space [Ab, p. 154]).

On the other hand, there exists a linear metric $G$-space $L$ which is not $G$ $\mathrm{AE}$ within the class of nonlocally convex spaces $\mathrm{Ca}$ and the class of countabledimensional spaces $[\mathrm{Ag}$. Therefore none of the hypotheses in the corollary below can be omitted.

Corollary 1.5. A metric linear $G$-space $L$ is a $G$-AE space if $L \in G$-ANE(0) and $L / G$ is countable dimensional.

A few comments about the proofs are in order. The class $\mathfrak{C}$ of all $G$-spaces satisfying the hypotheses of Theorem 1.4 is closed under the multiplication by $J=[0,1): \mathfrak{C} \supset \mathfrak{C} \times J$. In this situation the problem of an exact extension of a $G$-map $f: A \rightarrow X$ is solved by reducing to a less difficult problem of an approximate extension of a partial $G$-map.

Definition 1.6. A $G$-space $X$ is called an approximate absolute neighborhood $G$ extensor $(X \in G$-A-ANE), provided that for every open cover $\omega \in \operatorname{cov} X$ and every partial $G$-map $Z \hookleftarrow A \stackrel{f}{\rightarrow} X$ there exists a $G$-map $\tilde{f}: U \rightarrow X$, called an $\omega$-extension of $f$, defined on a $G$-neighborhood $U$ of $A$ such that $\operatorname{dist}\left(f,\left.\tilde{f}\right|_{A}\right) \prec \omega$.

The following theorem plays the key role in our exposition:

Theorem 1.7. Suppose that a class of metric $G$-spaces $\mathfrak{P}$ is closed under the multiplication by $J$. Then $\mathfrak{P} \subset G$-ANE if and only if $\mathfrak{P} \subset G$-A-ANE.

Implicitly this criterion with no group action being considered can be found in [Do], $\mathrm{Ma}$, [To. However, its fundamental role in the theory of absolute extensors becomes clear only now since:

$(\alpha)$ it is substantially easier to construct an approximate extension of a $G$-map than to get an exact one;

$(\beta)$ if the class $\mathfrak{P}$ of $G$-spaces is closed under the multiplication by $J$, then the problems of detection of $G$-ANE and approximation $G$-ANE are equivalent within the class $\mathfrak{P}$; and

$(\gamma)$ the concept of an approximate $G$-ANE fits well with different concepts and constructions in the theory of extensors.

Since the class $\mathfrak{C}$ of $G$-spaces is closed under the multiplication by $J$, it suffices by virtue of Theorem 1.2 to establish that $X \in G$-A-ANE, for every $X \in \mathfrak{C}$. This enables one to apply the concept of fiberwise trivial maps in the sense of Ancel $\mathrm{An}$, due to its close connection with $G$-A-ANE.

Proposition 1.8. If for every metric $G$-space $Z$ and for every partial $G$-map $Z \hookleftarrow$ $A \stackrel{\varphi}{\rightarrow} X$, the projection $\pi_{\varphi}: G_{\varphi} \rightarrow A$ of the graph $G_{\varphi} \subset Z \times X$ of $\varphi$ onto $A$ is fiberwise $G$-trivial inside the projection $p \rightleftharpoons \mathrm{pr}_{Z}: Z \times X \rightarrow Z$, then $X$ is $G$-A-ANE.

Proposition 1.8 is an easy consequence of Proposition 4.4.

\section{Preliminaries}

Hereafter we shall assume that all spaces (resp. maps) are metric (resp. continuous), unless otherwise stated. We shall denote the set of all open covers of $X$ by 
$\operatorname{cov} X$. The $\varepsilon$-neighborhood of $A \subset X, \varepsilon>0$, is the set

$$
\mathrm{N}(A ; \varepsilon) \rightleftharpoons\{x \in X \mid \operatorname{dist}(x, A)<\varepsilon\} .
$$

The body of a system of open sets $\omega$ is the set

$$
\cup \omega \rightleftharpoons \bigcup\{U \mid U \in \omega\}
$$

As always, $\omega \succ \omega_{1}$ denotes that the cover $\omega$ is a refinement of $\omega_{1}$. It is well-known that every cover $\sigma \in \operatorname{cov} X$ of a metric space $X$ admits a star refinement (the Stone theorem). Below we give a well-known and useful criterion for the star-refinability of covers:

Proposition 2.1. The cover $\sigma=\left\{S_{\lambda} \mid \lambda \in \Lambda\right\}$ is a star refinement of $\omega=\left\{W_{\beta} \mid \beta \in\right.$ $B\}$ if and only if for every $\lambda$ there exists $\beta=\beta(\lambda)$ such that $\bigcap_{\lambda \in \Lambda^{\prime}} S_{\lambda} \neq \emptyset, \Lambda^{\prime} \subset \Lambda$, implies that:

(1) $\bigcup_{\lambda \in \Lambda^{\prime}} S_{\lambda} \subset \bigcap_{\lambda \in \Lambda^{\prime}} W_{\beta_{\lambda}}$.

If $f, g: X \rightarrow Y$ are any maps, $A \subset Y$ and $\omega \in \operatorname{cov} Y$, then the condition of $\omega$-closeness of $f$ and $g$ will be denoted by $\operatorname{dist}(f, g) \prec \omega$ and $f \uparrow_{A}$ will denote the restriction of $f$ onto $A$.

Hereafter, we shall consider only compact groups. An action of $G$ on a space $X$ is a homeomorphism $T: G \rightarrow$ Aut $X$ of the group $G$ into the group Aut $X$ of all autohomeomorphisms of $X$ such that the map $G \times X \rightarrow X$, given by $(g, x) \mapsto$ $T(g)(x)=g x$, is continuous. A space $X$ with a fixed action of $G$ is called a $G$-space, and a map $f: X \rightarrow Y$ of $G$-spaces preserving an action of $G$ (i.e. $f(g \cdot x)=g \cdot f(x)$ ) is called a $G$-map. For a metric $G$-space $Z$, every $G$-map $\varphi: A \rightarrow X$, defined on a closed invariant subset $A \subset Z$ of $Z$, is called a partial $G$-map.

For a point $x \in X$, the isotropy subgroup of $x$, or the stabilizer of $x$, is defined as $G_{x}=\{g \in G \mid g x=x\}$, and the orbit of $x$ as $G(x)=\{g x \mid g \in G\}$. The space of all orbits is denoted by $X / G$ and the natural map $\pi: X \rightarrow X / G$, given by $\pi(x)=G(x)$, is called the orbit projection. The orbit space $X / G$ is equipped with the quotient topology, induced by $\pi$.

Definition 2.2. A $G$-space $X$ is said to be locally $G$-contractible, written as $X \in$ $G$-LC, if for every point $x$ and every $\epsilon>0$ there exist a point $x_{0} \in \mathrm{N}(x ; \varepsilon)$, a $G_{x_{0}}$-neighborhood $V, V \subset \mathrm{N}(x ; \varepsilon)$, of $x$ and a $G_{x_{0}}$-homotopy $F_{t}: V \rightarrow \mathrm{N}(x ; \varepsilon)$ such that

(2) $G_{x} \subset G_{x_{0}}$ and $G\left(x_{0}\right) \in G$-ANE; and

(3) $F_{0}=\mathrm{Id}, F_{1}(V)=x_{0}$ (i.e. $G_{x_{0}}$-neighborhood $V$ of $x$ is $G_{x_{0}}$-contractible to the point $x_{0}$ inside the neighborhood $\left.N(x ; \epsilon)\right)$.

Remark. For compact Lie groups $G$, each orbit $G\left(x_{0}\right)$ is $G$-ANE. Hence, by Theorem 1.2 , we can conclude that the definition of local $G$-contractibility stated above agrees with the one given in Chapter 1.

A space $D$ is said to be countable-dimensional if $D=\bigcup_{i=1}^{\infty} D_{i}$ and $D_{i}$ is zerodimensional, for every $i$. The following result follows easily from Lemma 3.3 of [An] and the openness of the orbit projection $\pi: A \rightarrow A / G$. 
Proposition 2.3. Let the orbit space $A / G$ of a closed $G$-set $A$ of the metric $G$ space $Z$ be countable-dimensional. Then for every sequence $\left\{\omega_{i}\right\}_{i \geq 1}$ of collections of open $G$-subsets in $Z$, covering $A$, there exists a sequence $\left\{\sigma_{i}\right\}_{i \geq 1}$ of collections of open $G$-subsets in $Z$ such that:

(4) $\sigma \rightleftharpoons \bigcup_{i=1}^{\infty} \sigma_{i}$ covers $A$;

(5) The collection $\sigma_{i}$ is disjoint; and

(6) $\sigma_{i} \succ \omega_{i}$, for every $i$.

\section{An APPROXimate CRITERION FOR EXTENDABILITy OF $G$-MAPS}

Let $A$ be a closed subset of a metric space $X$. An open cover $\omega$ of $Z \backslash A$ is said to be adjacent to $A$ if for every point $a \in A$ and its every neighborhood $U=U(a)$ in $X$ there exists a neighborhood $V(a) \subset U$ such that the star of $V(a)$ with respect to the cover $\omega$ lies in $U$. Such covers are usually called canonical covers $[\mathrm{BO}],[\mathrm{Hu}$, but we prefer the term 'adjacent' because of its stronger expressiveness.

In the product $X \times J$ of a metric $G$-space $X$ and $J=[0,1)$ we consider an open cover $\omega$, adjacent to the top level. The next theorem allows one to reduce the problem of the exact extension of partial maps to a less difficult problem of approximate extensions:

Theorem 3.1 (Approximate criterion for extendability). Let $\omega \in \operatorname{cov} X \times[0,1)$ be a cover which is adjacent to the top level $X \times\{1\}$. Then a partial $G$-map $Z \supseteq A \stackrel{f}{\rightarrow} X$ has a global [neighborhood] equivariant extension if and only if the partial G-map $Z \times J \supseteq A \times J \stackrel{f \times I d}{\longrightarrow} X \times J$ has an equivariant $\omega$-extension onto $Z \times J$ [onto a neighborhood of $A \times J$ ].

It follows immediately from Theorem 3.1 that $X \times J \in G$-A-ANE implies $X \in G$ ANE. This observation completes the proof of Theorem 1.8

Proof of Theorem 3.1. The nontrivial part of the proof is to establish the sufficiency. Thus, assuming the approximate extension property of $X \times J$ above, one needs to construct a $G$-extension of a given partial $G$-map $Z \supseteq A \stackrel{f}{\rightarrow} X$. By the hypothesis, the partial $G$-map $Z \times J \supseteq A \times J \stackrel{g}{\rightarrow} X \times J$, where $g=f \times \operatorname{Id}_{J}$, admits a $G$-map $\widetilde{g}: Z \times J \rightarrow X \times J$ [resp. $G$-map $\widetilde{g}: U \rightarrow X \times J]$, such that $\left(g, \widetilde{g}\left\lceil_{A \times J}\right) \prec \omega\right.$.

The following fact can be derived easily from the hypothesis that $\omega$ is adjacent to the top level and the continuity of $g$.

Claim 3.2. The $G$-map $d: A \times[0,1] \rightarrow X \times[0,1]$, given by the formulae $d \uparrow_{A \times J}=$ $\widetilde{g} \uparrow_{A \times J}$ and $d \uparrow_{A \times\{1\}}=f \times\{1\}$, is continuous.

We state the next straightforward fact without proof:

Claim 3.3. Suppose that a $G$-map $\alpha: F \cup W \rightarrow T$ is defined on the union of a closed $G$-subset $F$ and an open $G$-subset $W$ of a $G$-space $S$, such that $\alpha \uparrow_{F}$ and $\alpha \uparrow_{W}$ are continuous. Then there exists a closed $G$-subset $F_{1}, F \cup W \supseteq F_{1} \supseteq F$, of $S$ such that $\alpha \uparrow_{F_{1}}$ is continuous and $F \cap W \subseteq \operatorname{Int}\left(F_{1}\right)$.

Apply Claim 3.3 for $S \rightleftharpoons Z \times I, T \rightleftharpoons X \times I, F \rightleftharpoons A \times I \bigcup Z \times\{0\}, W \rightleftharpoons$ $Z \times J \quad[$ resp. $F \rightleftharpoons A \times I, W \rightleftharpoons U]$ and $\alpha \rightleftharpoons d \cup \widetilde{g}$. We get a closed $G$-subset $H$ of 
$Z \times I$, such that

$$
\begin{gathered}
A \times I \cup Z \times J \supseteq H \supseteq A \times I \cup Z \times\{0\}[\text { resp. } A \times I \cup U \supseteq H \supseteq A \times I], \\
A \times[0,1) \cup Z \times\{0\} \subseteq \operatorname{Int} H[\text { resp. } A \times[0,1) \subseteq \operatorname{Int} H]
\end{gathered}
$$

and $\alpha \uparrow_{H}$ is a continuous $G$-map.

Claim 3.4. There exists a sequence of open $G$-neighborhoods $V_{1} \supseteq V_{2} \supseteq \cdots, V_{i} \supseteq$ $\mathrm{Cl} V_{i+1}, \bigcap V_{i}=A$, and a monotone sequence of numbers $0=r_{0}<r_{1}<r_{2}<$ $\ldots, \lim r_{i}=1$, such that $V_{i} \times\left[0, r_{i}\right] \subseteq H$.

Let $\xi_{i}: Z \rightarrow\left[r_{i-2}, r_{i-1}\right], i \geq 2$, be a continuous real-valued function, constant on the orbits, and such that $\xi_{i}\left\lceil_{\mathrm{Bd} V_{i-1}} \equiv r_{i-2}\right.$ and $\xi_{i}\left\lceil_{\mathrm{Bd} V_{i}} \equiv r_{i-1}\right.$. Then the $G$ $\operatorname{map} \xi: Z \rightarrow[0,1]$, defined by $\xi\left\lceil Z \backslash V_{1}=0, \xi\left\lceil_{V_{i-1} \backslash V_{i}}=\xi_{i}\right.\right.$ for $i \geq 2$ and $\xi \uparrow_{A} \equiv \mathrm{Id}$, is continuous and $(v, \xi(v)) \in H$, for each $v \in Z \quad[$ resp. $(v, \xi(v)) \in H$, for each $\left.v \in V_{1}\right]$. The desired extension $\widehat{f}$ of the partial map $f$ is now given by the formula $\widehat{f}(v)=\alpha(v, \xi(v))$, where $v \in Z\left[\right.$ resp. $\left.v \in V_{1}\right]$.

\section{Fiberwise $G$-trivial Maps}

Consider the projection $p \rightleftharpoons \operatorname{pr}_{M}: M \times N \rightarrow M$ of the product of metric $G$ spaces onto the first factor $M$. Let the image $p(X)$ of a $G$-subset $X \subset M \times N$ (not necessarily closed) be contained in a $G$-subspace $Y \subset M$. Then we shall denote the restriction of $p$ onto $X$ by $\pi: X \rightarrow Y$. We do not require that a $G$-map $\pi$ be surjective.

We introduce some new concepts. A $G$-embedding $A \hookrightarrow B$ of $G$-subsets $A$ and $B$ of $M \times N$ is said to be fiberwise $G$-contractible inside the projection $p$, if there exist a $G$-map $g: p(A) \rightarrow B, p \circ g=\operatorname{Id}_{p(A)}$, and a $G$-homotopy $H_{t}: A \rightarrow B$ such that the following conditions $(1)-(3)$ are satisfied:

(1) $p \circ H_{t}=p$, for every $t \in I$ (i.e. $H_{t}$ is the fiberwise $G$-homotopy);

(2) $H_{0}=\mathrm{Id}_{A}$; and

(3) $H_{1}=g \circ p$ (the condition of factorizability of $H_{1}$ via the projection $p$ ).

Hereafter $\Omega$ shall denote the collection of all $G$-neighborhoods $\mathcal{U}$ of $X$ such that:

(4) $Y \subset p(\mathcal{U})$; and

(5) for every point $y \in Y$ there exists a neighborhood $O \rightleftharpoons O(y)$ of $y$ such that $O \times \operatorname{pr}_{N}\left(O^{\bullet} \cap X\right) \subset \mathcal{U}$. (We denote the product $C \times N=p^{-1}(C)$ by $C^{\bullet}$, where $C \subset M$.)

Let $\mathcal{U}, \mathcal{V} \in \Omega$ and $\mathcal{V} \subset \mathcal{U}$. We will say that a $G$-map $\pi=p \uparrow_{X}: X \rightarrow Y$ is fiberwise $G-\mathcal{U V}$-contractible inside the projection $p$, if there exists a $G$-neighborhood $W \subset p(\mathcal{V})$ of the $G$-set $Y$ such that the embedding $W^{\bullet} \cap \mathcal{V} \hookrightarrow W^{\bullet} \cap \mathcal{U}$ is fiberwise contractible inside the projection $p$.

We shall say that a map $\pi: X \rightarrow Y$ is fiberwise $G$-trivial inside the projection $p$, if for every $G$-neighborhood $\mathcal{U} \in \Omega$ there exists a small $G$-neighborhood $\mathcal{V} \in$ $\Omega, \mathcal{V} \subset \mathcal{U}$, for which the $G$-map $\pi: X \rightarrow Y$ is fiberwise $G-\mathcal{U V}$-contractible inside the projection $p$; locally fiberwise $G$-trivial inside the projection $p$, if for every $G$ neighborhood $\mathcal{U} \in \Omega$ there exist a $G$-neighborhood $\mathcal{V} \in \Omega, \mathcal{V} \subset \mathcal{U}$, and a collection $\sigma=\{O(y) \mid y \in Y\}$ of open (in $M) G$-sets, which covers $Y$ such that $p(\mathcal{V}) \supset \bigcup \sigma$ and $O(y)^{\bullet} \cap \mathcal{V} \hookrightarrow O(y)^{\bullet} \cap \mathcal{U}$ is fiberwise $G$-contractible inside $p$, for every $y \in Y$. 
Proposition 4.1. Suppose that there is a sequence of $G$-neighborhoods $\mathcal{V}_{1} \supset \mathcal{V}_{2} \supset$ $\mathcal{V}_{3} \supset \ldots$ from $\Omega$ and that a $G$-space $Y$ is contained in the union $\bigcup_{i=1}^{\infty} W_{i}$ of open $G$-subsets of $M$. Suppose that for every $i \geq 1$, the following conditions are satisfied:

(6) $p\left(\mathcal{V}_{i+1}\right) \supset W_{i}$; and

(7) the embedding $W_{i}^{\bullet} \cap \mathcal{V}_{i+1} \hookrightarrow W_{i}^{\bullet} \cap \mathcal{V}_{i}$ is fiberwise contractible inside $p$.

Then $\mathcal{V} \rightleftharpoons \bigcup_{i=1}^{\infty} W_{i}^{\bullet} \cap \mathcal{V}_{i+1} \in \Omega$ and the projection $\pi$ is fiberwise $G$ - $\mathcal{V}_{1} \mathcal{V}$ contractible inside $p$.

This proposition is an equivariant version of Lemma 3.6 of [An, p. 11] and its proof is analogous, and is based on the openness and perfectness of the orbit projection.

We now present a condition on a $G$-space $X$, which guarantees the local fiberwise $G$-triviality of the graph projection.

Proposition 4.2. Let $Z \hookleftarrow A \stackrel{\varphi}{\longrightarrow} X$ be a partial $G$-map onto a $G$-LC-space $X$ and $G_{\varphi} \rightleftharpoons\{(a, \varphi(a)) \mid a \in A\} \subset Z \times X$ be graph of $\varphi$. Then the projection $\pi: G_{\varphi} \rightarrow A$ of the graph $G_{\varphi}$ onto $A$ is locally fiberwise $G$-trivial inside the projection $p: Z \times X \rightarrow$ $Z$.

Proof. It is easy to check that the collection $\Omega$ of all $G$-neighborhoods of $G_{\varphi}$ in $Z \times X$ satisfies properties (4)-(5) for $p: Z \times X \rightarrow Z$. Next we fix a closed $G$-neighborhood $\mathcal{U}$ of $G_{\varphi}$. We should find a $G$-neighborhood $\mathcal{V} \in \Omega, G_{\varphi} \subset \mathcal{V} \subset \mathcal{U} \subset Z \times X$, such that:

(a) For every point $a_{0} \in A$ there exists a $G$-neighborhood $O=O\left(a_{0}\right) \subset Z$, for which $O \subset p(\mathcal{V})$ and the embedding $O^{\bullet} \cap \mathcal{V} \hookrightarrow O^{\bullet} \cap \mathcal{U}$ is fiberwise $G$-contractible (here $O^{\bullet} \rightleftharpoons O \times X$ ).

We shall assume that some invariant metrics are given on $X$ and $Z[\mathrm{~Pa}]$. We shall investigate a multivalued map

$\Phi: A \rightsquigarrow \mathbb{R}^{+}, \Phi(a)=\{r>0 \mid \mathrm{N}(G(\bar{a}) ; r) \subset \mathcal{U}\} \subset \mathbb{R}^{+}$, where $\bar{a} \rightleftharpoons(a, \varphi(a)) \in G_{\varphi}$.

It is easy to see that $\Phi$ is lower semi-continuous, convex-and-closed-valued, and is constant on the orbits.

Since the group $G$ acts trivially on $J$, we can apply the Dowker theorem [RS] to the induced multivalued map $\widetilde{\Phi}: A / G \rightsquigarrow \mathbb{R}^{+}$and obtain a selection $r: A \rightarrow(0, \infty)$ of $\Phi$ which is constant on each orbit.

Next, we need an auxiliary fact, based on the hypothesis that $X \in G$-LC.

Lemma 4.3. For every $a \in A$, there exists a $G$-neighborhood $\mathcal{O}$ of $\bar{a}=(a, \varphi(a))$ such that $\mathcal{O} \subset \mathrm{N}(G(\bar{a}) ; r(a))$ and the natural embedding $\mathcal{O} \hookrightarrow \mathrm{N}(G(\bar{a}) ; r(a))$ is fiberwise $G$-contractible.

Proof. For $x=\varphi(a) \in X$ and $\varepsilon=r(a)>0$, there exist $x_{0} \in \mathrm{N}(x ; \varepsilon)$, a $G_{x_{0}}$ neighborhood $V, V \subset \mathrm{N}(x ; \varepsilon)$, of $x$ and a $G_{x_{0}}$-homotopy $F_{t}: V \rightarrow \mathrm{N}(x ; \varepsilon)$ such that the conditions (2)-(3) of Definition [2.2 are fulfilled. Since $G\left(x_{0}\right) \in G$-ANE, the natural $G$-map $f: G(a) \rightarrow G\left(x_{0}\right), f(g \cdot a)=g \cdot x_{0}$, can be extended to a $G$-map $\widehat{f}: U(a) \rightarrow G\left(x_{0}\right)$ defined on a $G$-neighborhood $U(a)$ of $G(a)$.

It is not difficult to check that $S \rightleftharpoons \widehat{f}^{-1}\left(x_{0}\right)$ is a $G_{x_{0}}$-set of $Z, U(a)$ coincides with $G \times{ }_{G_{x_{0}}} S$ and $\mathcal{O}(\bar{a}) \rightleftharpoons G \cdot(S \times V)$ is a $G$-neighborhood of $G(\bar{a})$ in $Z \times X$. The reader can check that the formula $\Phi_{t}(g \cdot(s, v))=g \cdot\left(s, F_{t} v\right), s \in S, v \in V, g \in G$, correctly 
defines a $G$-homotopy $\Phi_{t}: \mathcal{O}(\bar{a}) \rightarrow Z \times X$, which is a fiberwise $G$-contraction. Reducing the $G$-neighborhood $U(a)$ if necessary, we can choose $\mathcal{O}(\bar{a})$ as the desired $G$-neighborhood $\mathcal{O}$.

We now continue with the proof of Proposition 4.2. Consider yet another multivalued map

$$
\begin{gathered}
\Psi: A \rightsquigarrow \mathbb{R}^{+}, \Psi(a)=\{t>0 \mid r(a) \geq t, \text { and the natural embedding } \\
\mathrm{N}(G(\bar{a}) ; t) \hookrightarrow \mathrm{N}(G(\bar{a}) ; r(a)) \text { is fiberwise } G \text {-contractible }\} \subset \mathbb{R}^{+},
\end{gathered}
$$

which is also easily seen to be lower semi-continuous, convex-and-closed-valued, and is constant on the orbits. We again invoke the Dowker theorem and find a continuous $G$-selection $t: A \rightarrow(0, \infty)$ of $\Psi$ such that $t(a) \leq r(a)$.

By virtue of the continuity of $\varphi$ and $t$, it is possible to choose a $G$-neighborhood $W\left(a_{0}\right)$ of $a_{0} \in A$ such that:

(b) For every $a \in W\left(a_{0}\right) \cap A, \mathrm{~N}(G(\bar{a}) ; t(a) / 2) \subset \mathrm{N}\left(G\left(\bar{a}_{0}\right) ; t\left(a_{0}\right)\right)$, where $\bar{a}_{0}=$ $\left(a_{0}, \varphi\left(a_{0}\right)\right)$.

The family of $G$-neighborhoods $\sigma \rightleftharpoons\{W(a) \subset Z \mid a \in A\}$ covers $A$. As $Z / G$ is paracompact, there exists a family $\sigma^{\prime}=\left\{W^{\prime}(a) \subset Z \mid a \in A\right\}$ of $G$-neighborhoods, covering $A$, which is star refinement of $\sigma$. By Proposition 2.1, it follows that:

(c) $\bigcap_{i=0}^{n} W^{\prime}\left(a_{i}\right) \neq \emptyset$ implies $\bigcup_{i=0}^{n} W^{\prime}\left(a_{i}\right) \subset \bigcap_{i=0}^{n} W\left(z_{a_{i}}\right) \subset W\left(z_{a_{0}}\right)$.

We associate to every point $a \in A$, a point $z_{a} \in A$ such that $\operatorname{St}_{\sigma^{\prime}}(a) \rightleftharpoons$ $\bigcup_{W^{\prime}\left(a^{\prime}\right)} W^{\prime}\left(a^{\prime}\right) \subset W\left(z_{a}\right)$. Finally, the desired $G$-neighborhood $\mathcal{V}$ is defined by $a \in W^{\prime}\left(a^{\prime}\right)$

$$
\mathcal{V} \rightleftharpoons \bigcup_{a \in A} \mathrm{~N}(G(\bar{a}) ; t(a) / 2) \cap\left(W^{\prime}(a)\right)^{\bullet} .
$$

It is clear that $G_{\varphi} \subset \mathcal{V} \subset \mathcal{U}$. To verify (a) we should prove the following properties of the $G$-neighborhood $O \rightleftharpoons W^{\prime}\left(a_{0}\right)$ of $a_{0} \in A$ :

(d) $O \bullet \cap \mathcal{V} \subset \mathrm{N}\left(G\left(\bar{z}_{a_{0}}\right) ; t\left(z_{a_{0}}\right)\right)$; and

(e) $O^{\bullet} \cap \mathrm{N}\left(G\left(\bar{z}_{a_{0}}\right) ; r\left(z_{a_{0}}\right)\right) \subset \mathcal{U}$, where $\bar{z}_{a_{0}}=\left(z_{a_{0}}, \varphi\left(z_{a_{0}}\right)\right) \in Z \times X$.

Property (e) is evident as $\bigcup_{a \in A} \mathrm{~N}(G(\bar{a}) ; r(a)) \subset \mathcal{U}$. To prove (d), pick a point $z \in O$. It follows from the explicit formulae for $\mathcal{V}$ and $O^{\bullet}$ that:

(f) $G(z)^{\bullet} \cap \mathcal{V} \subset \bigcup_{a \in \Lambda} \mathrm{N}(G(\bar{a}) ; t(a) / 2) \cap\left(W^{\prime}(a)\right)^{\bullet}$, where $\Lambda=\left\{a \in A \mid z \in W^{\prime}(a)\right\}$.

Since $z \in \bigcap_{a \in \Lambda} W^{\prime}(a)$ and $a_{0} \in \Lambda$ (as $z \in O=W^{\prime}\left(a_{0}\right)$ ), we have, by (c), that $\bigcup_{a \in \Lambda} W^{\prime}(a) \subset W\left(z_{a_{0}}\right)$. After invoking (b) the last inclusion implies

$$
\bigcup_{a \in \Lambda} \mathrm{N}(G(\bar{a}) ; t(a) / 2) \subset \mathrm{N}\left(G\left(\bar{z}_{a_{0}}\right) ; t\left(z_{a_{0}}\right)\right) .
$$

Since $t: A \rightarrow(0, \infty)$ is a $G$-selection of $\Psi, \mathrm{N}\left(G\left(\bar{z}_{a_{0}}\right) ; t\left(z_{a_{0}}\right)\right)$ is fiberwise $G$ contractible inside $\mathrm{N}\left(G\left(\bar{z}_{a_{0}}\right) ; r\left(z_{a_{0}}\right)\right)$. Therefore $O^{\bullet} \cap \mathcal{V} \hookrightarrow O^{\bullet} \cap \mathcal{U}$ is a fiberwise $G$-contractible embedding and this completes the proof of Proposition 4.2.

In conclusion we shall strengthen Proposition 1.8, which establishes the connection between fiberwise $G$-trivial maps and $G$-A-ANE. 
Proposition 4.4. Let $X$ be a closed subset of $G$-ANE-space $Z$. If the projection $\pi_{\varphi}: G_{\varphi} \rightarrow X$ of the graph $G_{\varphi} \subset Z \times X$ of the partial $G$-map $Z \hookleftarrow X \stackrel{\varphi \operatorname{Id}_{X}}{\longrightarrow} X$ is fiberwise $G$-trivial inside the projection $p \rightleftharpoons \operatorname{pr}_{Z}: Z \times X \rightarrow Z$, then $X \in G$-A-ANE.

Proof. We shall prove that for every cover $\omega=\left\{W_{\gamma} \mid \gamma \in \Gamma\right\} \in \operatorname{cov} X$ there exists a $G$-map $\widetilde{\varphi}: W \rightarrow X$, defined on a $G$-neighborhood $W$ of $X$ in $Z$, such that $\operatorname{dist}\left(\varphi, \widetilde{\varphi}\left\lceil_{X}\right) \prec \omega\right.$. When this is done, then $X \in G$-A-ANE, due to the following simple fact:

Lemma 4.5. Let $Z$ be a $G$-ANE space and suppose that a partial $G$-map $Z \hookleftarrow$ $X \stackrel{\varphi \operatorname{Id}_{X}}{\longrightarrow} X$ has a neighborhood $\omega$-G-extension, for every $\omega \in \operatorname{cov} X$. Then $X \in G$ A-ANE.

Consider a cover $\omega^{\prime}=\left\{W_{\beta}^{\prime}\right\} \in \operatorname{cov} X$ which is star refinement of $\omega$. It follows by Proposition 2.1 that there exists a map of the index sets $\beta \longmapsto \gamma=\gamma(\beta)$, such that the property 2.1(1) holds. In addition, consider an open system $\sigma=\left\{S_{\lambda}\right\}$ in $Z$, covering $X$ and such that $\left\{\varphi\left(S_{\lambda} \cap X\right)\right\} \succ \omega^{\prime}$. Let $\varphi\left(S_{\lambda} \cap X\right) \subset W_{\beta=\beta(\lambda)}^{\prime} \subset W_{\gamma=\gamma(\beta)}$.

Since the map $\pi_{\varphi}: G_{\varphi} \rightarrow X$ is fiberwise $G$-trivial inside $p: Z \times X \rightarrow Z$, it follows that for the neighborhood $\mathcal{U} \rightleftharpoons \bigcup_{\lambda} S_{\lambda} \times W_{\beta(\lambda)}^{\prime}$ of the graph $G_{\varphi}$, there exist a $G$ neighborhood $W, Z \supset W \supset X$, and a $G$-neighborhood $\mathcal{V}, G_{\varphi} \subset \mathcal{V} \subset \mathcal{U}$, such that $p(\mathcal{V}) \supset W$ and $W^{\bullet} \cap \mathcal{V}$ is fiberwise $G$-contractible inside $W^{\bullet} \cap \mathcal{U}$.

Consequently, there exists a $G$-map $\widetilde{\varphi}: W \rightarrow X$ such that $(x, \widetilde{\varphi}(x)) \in \mathcal{U}$, for every $x \in X$. We will show that $\widetilde{\varphi}$ is in fact the desired map. For $x_{0} \in X$ let $\Lambda^{\prime}$ be the set $\left\{\lambda \mid x_{0} \in S_{\lambda}\right\} \neq \varnothing$.

It is easy to see that $x_{0}^{\bullet} \cap \mathcal{U}=\bigcup_{\lambda \in \Lambda^{\prime}} x_{0} \times W_{\beta(\lambda)}^{\prime}=x_{0} \times\left(\bigcup_{\lambda \in \Lambda^{\prime}} W_{\beta(\lambda)}^{\prime}\right)$ and hence, by virtue of Proposition $2.1 x_{0}^{\bullet} \cap \mathcal{U} \subset x_{0} \times \bigcap_{\lambda \in \Lambda^{\prime}} W_{\gamma(\beta(\lambda))}$. Since $\left(x_{0}, \widetilde{\varphi}\left(x_{0}\right)\right) \in x_{0}^{\bullet} \cap \mathcal{U}$, it follows that $\widetilde{\varphi}\left(x_{0}\right) \in \bigcap_{\lambda \in \Lambda^{\prime}} W_{\gamma(\beta(\lambda))}$. Hence for every $\lambda \in \Lambda^{\prime}, \varphi\left(x_{0}\right)$ and $\widetilde{\varphi}\left(x_{0}\right)$ are contained in $W_{\gamma(\beta(\lambda))} \in \omega$.

\section{Proof of Theorem 1.4}

Consider the situation from the beginning of Chapter 4. This in particular means that $X \subset M \times N$ and $Y \subset N$ are $G$-subsets of metric $G$-spaces and $p(X) \subset Y$ where $p \rightleftharpoons \operatorname{pr}_{M}: M \times N \rightarrow M$ is the projection onto the first factor $M$. Let $\Omega$ be a collection of all $G$-neighborhoods of $X$ satisfying conditions (4)-(5) from Chapter 4.

Theorem 5.1. Let $\pi: X \rightarrow Y$ be a restriction of the projection $p \rightleftharpoons \operatorname{pr}_{M}: M \times$ $N \rightarrow M$ onto $X$. Let the orbit space $Y / G$ of the $G$-space $Y$ be countable-dimensional and the projection $\pi: X \rightarrow Y$ a locally fiberwise $G$-trivial inside $p$. Then the projection $\pi$ is fiberwise $G$-trivial inside $p$.

Proof. We fix a neighborhood $\mathcal{V}_{1} \in \Omega$ of the $G$-space $X$ in $M \times N$. Since $\pi$ is locally fiberwise $G$-trivial inside $p$, there exist a family $\sigma_{1}=\left\{O_{1}(y) \mid y \in Y\right\}$ of open $G$-sets in $M$, which covers $Y$, and a $G$-neighborhood $\mathcal{V}_{2} \subset \mathcal{V}_{1}, \mathcal{V}_{2} \in \Omega$, such that $p\left(\mathcal{V}_{2}\right) \supset \bigcup \sigma_{1}$ and the embedding $\left(O_{1}(y)\right)^{\bullet} \cap \mathcal{V}_{2} \hookrightarrow\left(O_{1}(y)\right)^{\bullet} \cap \mathcal{V}_{1}$ is fiberwise $G$-contractible inside $p$, for every $y \in Y$. 
Analogously, for $j \geq 3$, one can construct $G$-neighborhoods $\mathcal{V}_{j} \in \Omega, \mathcal{V}_{j} \subset \mathcal{V}_{j-1}$, and a collection $\sigma_{j-1}=\left\{O_{j-1}(y) \mid y \in Y\right\}$ of open $G$-sets in $M$, covering $Y$, such that:

1) $\sigma_{j-1} \succ \sigma_{j-2}$; and

2) $p\left(\mathcal{V}_{j}\right) \supset \bigcup \sigma_{j-1}$ and the embedding $\left(O_{j-1}(y)\right)^{\bullet} \cap \mathcal{V}_{j} \hookrightarrow\left(O_{j-1}(y)\right)^{\bullet} \cap \mathcal{V}_{j-1}$ is fiberwise $G$-contractible inside $p$, for every $y \in Y$.

Since $Y / G$ is countable-dimensional, Proposition 2.3 implies that

$$
Y \subset \bigcup_{j=1}^{\infty} \bigcup_{\lambda \in \Lambda_{j}} D_{j}(\lambda),
$$

where $\left\{D_{j}(\lambda) \mid \lambda \in \Lambda_{j}\right\}$ is a collection of disjoint open $G$-sets in $M$ which refines $\sigma_{j}$. We denote by $W_{j}=\bigcup_{\lambda \in \Lambda_{j}} D_{j}(\lambda)$, for $j \geq 1$.

It is evident that $p \mathcal{V}_{j+1} \supset W_{j}$, for every $j \geq 1$ and the embedding $W_{j}^{\bullet} \cap \mathcal{V}_{j+1} \hookrightarrow$ $W_{j}^{\bullet} \cap \mathcal{V}_{j}$ is fiberwise $G$-contractible inside $p$. Therefore conditions (6) and (7) from from Chapter 4 are satisfied. By virtue of Proposition 4.1, $\mathcal{V} \rightleftharpoons \bigcup_{j=1}^{\infty} W_{j}^{\bullet} \cap \mathcal{V}_{j+1} \in \Omega$ and $\pi$ is fiberwise $G-\mathcal{V}_{1} \mathcal{V}$-contractible inside $p$. This completes the proof.

Proof of Theorem 1.4. Since the class of spaces, which satisfy the hypotheses of Theorem 1.4, is closed under the multiplication by $[0,1)$, it suffices, by Theorem 3.1, to prove that $X \in G$-LC implies $X \in G$-A-ANE.

Take a closed embedding of $X$ into a linear normed space $L[$ Bo]. By $Z$ we denote a linear normed space $\mathrm{C}(G, L)$ of all continuous maps $f: G \rightarrow L$ endowed by the action of $G:(g \cdot f)(h)=f\left(g^{-1} \cdot h\right)$, where $f \in \mathrm{C}(G, L), g, h \in G$. By $\mathrm{Mx}$. Theorem 3], $Z \in G$-ANE. Consider a closed $G$-embedding $\varphi: X \hookrightarrow Z$ of $X$ into $Z$, given by the formula $\varphi(x)(g)=g^{-1} \cdot x$, where $x \in X$ and $g \in G$, and define $G_{\varphi} \subset Z \times X, \pi_{\varphi}: G_{\varphi} \rightarrow X$ and $p: Z \times X \rightarrow Z$ as in Proposition 4.4.

Since $X \in G$-LC, it follows by Proposition 4.2 that $\pi_{\varphi}$ is a locally fiberwise $G$-trivial projection inside $p$. Since $X$ has a countable-dimensional orbit space, all hypotheses of Theorem 5.1 are satisfied and consequently $\pi_{\varphi}$ is a fiberwise $G$-trivial projection inside $p$. Finally it follows by Proposition 4.4 that $X \in G$-A-ANE.

\section{ACKNOWLEDGEMENTS}

The first author was supported in part by the INTAS grant No. 96-0712. The second author was supported in part by the Ministry of Science and Technology of the Republic of Slovenia grant No. J1-0885-0101-98. We thank the referee for very close reading of the manuscript and several comments and suggestions.

\section{REFERENCES}

[Ab] H. Abels, A universal proper G-space, Math. Z. 159 (1978), 143-158. MR 58:18504

[AG] D. F. Addis and J. H. Gresham, A class of infinite-dimensional spaces I, Dimension theory and Aleksandroff's problem, Fund. Math. 101 (1978), 195-205. MR 80b:54041

[Ag] S. M. Ageev, An equivariant Dugundji theorem, Uspehi Mat. Nauk 45:5 (1990), 116-117 (in Russian); Engl. transl. in: Russ. Math. Surv. 45:5 (1990), 219-220. MR 92h:54026

[An] F. D. Ancel, The role of countable dimensionality in the theory of cell-like relations, Trans. Amer. Math. Soc. 287 (1985), 1-40. MR 86b:54012

[Bo] K. Borsuk, Theory of Retracts, Monogr. Matem. 44, PWN, Warsaw, 1967. MR 35:7306

[Br] G. E. Bredon, Introduction to Compact Transformation Groups, Pure and Appl. Math. 46, Academic Press, New York-London, 1972. MR 54:1265 
[Ca] R. Cauty, Un espace metrique lineaire qui n'est pas un retracte absolu, Fund. Math. 146 (1994), 85-99. MR 95j:54022

[Do] C. H. Dowker, Homotopy extension theorems, Proc. London Math. Soc. 6 (1956), 100-116. MR 17:518f

[Ha] W. E. Haver, Locally contractible spaces that are absolute neighborhood retracts, Proc. Amer. Math. Soc. 40 (1973), 280-284. MR 48:9645

[Hu] S.-T. Hu, Theory of Retracts, Wayne State Univ. Press, Detroit, 1965. MR 31:6202

[Ja] J. Jaworowski, Extensions of G-maps and Euclidean G-retracts, Math. Z. 146 (1976), 143148. MR 52:15351

[Ma] S. Mardešić, Approximate polyhedra, resolutions of maps and shape fibrations, Fund. Math. 114 (1981), 53-78. MR 83d:55016

[Mx] C. N. Maxwell, Homeomorphisms of topological transformation groups into function spaces, Duke Math. J. 33 (1966), 567-574. MR 34:3563

[Mu] T. Murayama, On G-ANE's and their G-homotopy types, Osaka J. Math. 20 (1983), 479512. MR 85f:57022

[Pa] R. S. Palais, The classification of G-spaces, Memoirs Amer. Math. Soc. 36 (1960). MR 31:1664

[RS] D. Repovš and P. V. Semenov, Continuous Selections of Multivalued Mappings, Mathematics and Its Appl. 455, Kluwer, Dordrecht, 1998. MR 2000a:54002

[To] H. Toruńczyk, Concerning locally homotopy negligible sets and characterization of $l_{2}$ manifolds, Fund. Math. 101 (1978), 93-110. MR 80g:57019

[Vi] G. Villalobos, Inversion of the theorem on the existence of slices, Vestnik MSU (1) (2000), 26-29 (in Russian).

Department of Mathematics, Brest State University, Brest, Belarus 224011

E-mail address: ageev@highmath.brsu.brest.by

Institute of Mathematics, Physics and Mechanics, University of Ljubljana, P.O. Box 2964, LuUbluana, Slovenia 1001

E-mail address: dusan.repovs@uni-lj.si 